\title{
Organizações policiais frente à pandemia: sensemaking, liderança e discricionariedade
}

\author{
Gustavo Matarazzo 12 \\ Alan Fernandes 23 \\ Rafael Alcadipani 2 \\ 1 Instituto Federal de Educação, Ciência e Tecnologia de São Paulo, São Paulo / SP - Brasil \\ 2 Fundação Getulio Vargas / Escola de Administração de Empresas de São Paulo, São Paulo / SP - Brasil \\ 3 Polícia Militar do Estado de São Paulo, São Paulo / SP - Brasil
}

\begin{abstract}
O enfrentamento da pandemia da COVID-19 desponta como um dos maiores desafios recentes impostos à humanidade. Dentre as instituições públicas especialmente acionadas, as polícias estão posicionadas na linha de frente. A partir de suas características organizacionais e profissionais, discute-se estratégias de enfrentamento à pandemia por meio do desenvolvimento de novas habilidades organizacionais que propiciem alterar suas lógicas de funcionamento de "guerra contra o crime" para ações humanitárias. Assim, aponta-se a necessidade de coordenação sistêmica dos órgãos policiais, tanto entre em si, como no conjunto das demais medidas de enfrentamento. Nesses termos, a partir da análise de experiências diversas, propõe-se a formação de uma articulação interorganizacional fundamentada na criação de elementos linguísticos e materiais, com o compartilhamento de mapas estratégicos e planos informacionais. Para tanto, conclui-se que a revisão de protocolos de atuação das polícias em emergências de saúde pública, a incluir o Plano de Resposta em Emergências em Saúde Pública, do Ministério da Saúde, tem a capacidade de prover avanços nessa questão.
\end{abstract}

Palavras-chave: COVID-19; polícia; sensemaking.

\section{Organizaciones policiales frente a la pandemia: sensemaking, liderazgo y discrecionalidad}

Hacer frente a la pandemia de COVID-19 se está convirtiendo en uno de los mayores desafíos recientes que enfrenta la humanidad. Entre las instituciones públicas especialmente activadas, la policía está posicionada en primera línea. En función de sus características organizativas y profesionales, las estrategias para hacer frente a la pandemia se discuten mediante el desarrollo de nuevas habilidades organizativas que les permitan cambiar su lógica operativa de "guerra contra el crimen" a acciones humanitarias. Por lo tanto, señala la necesidad de una coordinación sistémica de los cuerpos policiales, tanto entre ellos como en el conjunto de otras medidas de afrontamiento. En estos términos, a partir del análisis de diferentes experiencias, se propone formar una articulación interorganizacional basada en la creación de elementos lingüísticos y materiales, con el intercambio de mapas estratégicos y planes informativos. Por lo tanto, se concluye que la revisión de los protocolos de desempeño policial en emergencias de salud pública, incluido el Plan de Respuesta a Emergencias de Salud Pública, del Ministerio de Salud, tiene la capacidad de proporcionar avances en este tema.

Palabras clave: COVID-19; Policía; sensemaking.

\section{Police institutions in the face of the pandemic: sensemaking, leadership, and discretion}

Coping with the COVID-19 pandemic is emerging as one of the greatest recent challenges facing humanity. The police forces are at the front line, among the public institutions specially activated in this emergency. Based on police forces' organizational and professional characteristics, this study discusses strategies to deal with the pandemic through the development of new organizational skills that allow them to change their operating logic from "war against crime" to humanitarian actions. The research points out the need for systemic coordination of police bodies, both among themselves and in the set of other coping measures. In these terms, from the analysis of different experiences, it is proposed to form inter-organizational coordination based on the creation of linguistic and material elements, with the sharing of strategic maps and informational plans. In conclusion, the review of police performance protocols in public health emergencies, including the Public Health Emergency Response Plan of the Ministry of Health, has the capacity to improve its response in the face of the COVID-19 pandemic. Keywords: COVID-19; police; sensemaking. 


\section{INTRODUÇÃO}

A pandemia da COVID-19 desponta como um dos maiores desafios da história recente da humanidade. A disseminação do vírus tem levado as instituições do Estado, em especial aquelas diretamente envolvidas com a gestão de populações, a implementarem o isolamento social com o objetivo de atenuar o contágio. Neste contexto, evoca-se o papel das forças de segurança pública na garantia da governabilidade, especialmente no cumprimento da medida compulsória do isolamento social, e na segurança do pessoal e dos equipamentos de saúde, diretamente relacionados à profilaxia da doença. No Brasil, a polícia tem atuado na investigação de furtos e roubos de equipamentos de proteção pessoal contra o vírus e de testes para a doença; na averiguação de fraude de equipamentos e de processos licitatórios; na fiscalização do cumprimento das regras de funcionamento das atividades econômicas, e até mesmo na prisão de pessoas que desrespeitam as determinações.

A polícia é uma das categorias da linha de frente do combate à pandemia mais vulneráveis ao contágio. Em Nova York, por exemplo, grande foco da doença, até o dia 7 de abril de 2020, quase $20 \%$ do contingente policial havia sido contaminado pelo vírus e doze membros haviam chegado a óbito. No Brasil, ainda não há levantamentos a respeito deste fato. No entanto, uma pesquisa realizada pela Fundação Getúlio Vargas em parceria com o Fórum Brasileiro de Segurança Pública divulgada no mês de maio de 2020 aponta que, em São Paulo, 59,7\% dos policiais civis e militares sentem medo de contrair ou ter algum familiar contaminado pelo novo coronavírus e, o pior, apenas $1 / 3$ dos policiais do Brasil declararam ter recebido equipamento de proteção individual e treinamento para lidar com a pandemia em seu trabalho cotidiano.

Organizações policiais são marcadas por fortes conteúdos cognitivos internos, por cultura organizacional bastante peculiar e culturas ocupacionais específicas (Rubinstein, 1973; Manning, 1977). O cenário atual da pandemia da COVID-19 impõe uma mudança de perspectiva da atuação policial, a qual privilegia o modelo de atuação de "combate ao crime". Julga-se necessário, neste contexto, discutir sobre a adoção de modelos de gestão interna e de práticas mais adequados e direcionados às ações humanitárias (Alcadipani, Cabral, Fernandes, \& Lotta, 2020). Organizações de "linha de frente", como as polícias, precisam urgentemente implementar mudanças organizacionais para o enfrentamento da pandemia, levando em conta uma multiplicidade de fatores: biológicos, sociais e político aqui evocados.

Assim, o presente artigo discute estratégias de enfrentamento à pandemia, por parte das polícias, e propõe o desenvolvimento de novas habilidades organizacionais, impulsionando os esforços para a elaboração de um modelo de atuação, que coordene diferentes agências e garanta um novo sentido à atuação das corporações policiais. Para tanto, estruturamos este artigo com uma seção, após a introdução, que foca a coordenação sistêmica das ações de diferentes agências. Em seguida, discutimos a necessidade de alterar a produção de sentidos das polícias, como o foco específico em questões de discricionariedade e emoção. Por fim, discutimos sugestões de elementos práticos que as polícias podem adotar para lidar com a pandemia.

\section{COORDENAÇÃO SISTÊMICA E INCLUSÃO DAS POLÍCIAS NO ENFRENTAMENTO À PANDEMIA}

Nos estudos de burocracia, comparativamente a outras organizações do Estado, as polícias constituem exemplos evidentes de insulamento, motivado por conteúdos cognitivos específicos que caracterizam maneiras próprias de atuação (Giacomantonio, 2014; Mastrofski \& Willis, 2010; Monjardet, 1998). No Brasil, por exemplo, as polícias civil e militar têm extrema dificuldade em articular ações em 
conjunto e compartilhar informações, o que denota a carência de instrumentos que promovam uma atuação sistêmica das diferentes agências de polícia, bem como de um arranjo mais amplo que conecte diversas áreas estatais. No contexto atual, somam-se à dificuldade da coordenação interagências no Brasil os obstáculos intrínsecos e próprios do combate à pandemia.

Contextos como o da pandemia atual exigem decisões rápidas e variadas, o que implica recursos e orçamentos urgentes para a atuação das organizações (Comes, 2016). As respostas a contextos como este costumam encontrar problemas nos processos comunicacionais, no exercício de autoridade e no desenvolvimento de coordenações (Quarantelli, 1988). Além do mais, ao considerarmos os processos comunicacionais em organizações policiais, identificamos que seus sistemas de tecnologia de informação são difusos e, de modo geral, não compartilham os mesmos bancos de dados (Manning, 2008). No Brasil, o diálogo entre os sistemas de dados das polícias Militar, Civil e Federal é ainda mais raro.

Para que as organizações atuem de maneira coordenada, é importante que os ocupantes dos cargos diretivos compartilhem um mesmo mapa estratégico do contexto em questão. Os networkings dos diretores e comandantes das organizações precisam ser acionados no sentido de contribuir para criar e fortificar os fluxos de informações por meio de estruturas burocráticas adaptáveis (Stephenson, 2005). Sabemos que as organizações policiais são fortemente apegadas a hábitos e costumes (Manning, 2007, 2008), o que pode criar obstáculos à celeridade necessária do momento. De acordo com isso, acreditamos que seja muito importante a atuação de analistas organizacionais, tendo em vista a elaboração de ambientes e dispositivos propícios para a tomada de decisão conjunta.

Em resumo, a coordenação de ações interorganizacionais é um aspecto fundamental a ser desenvolvido entre as diferentes instituições governamentais para enfrentar a pandemia. No Brasil, raramente os governos percebem que as polícias podem contribuir no combate à pandemia diariamente analisando os fluxos populacionais, já que as polícias possuem instrumentos para isso, e dando suporte aos profissionais de saúde na organização das unidades de atendimento. A inclusão das polícias no sistema de gerenciamento da pandemia depende da disponibilização de fluxos de informações similares das demais organizações que estão lidando com a doença.

Diante disso, a construção de sentidos (sensemaking) das variadas organizações que atuam no combate à pandemia deve compartilhar um plano informacional que favoreça a interoperabilidade entre elas. No caso das polícias, esse esforço é ainda mais desafiador, pois requer a produção de novos sentidos, como discutiremos a seguir.

\section{PRODUÇÃO DE SENTIDOS DAS POLÍCIAS PARA A PANDEMIA}

Comumente, atribui-se uma lógica racional à tomada de decisão por parte das organizações policiais. No entanto, dada a variedade de contextos em que as polícias atuam, surgem racionalidades específicas que não são evidentes nem autoexplicáveis. Isto é, a "versão racional" existe, contudo, ela é estabelecida situacionalmente, conforme os contextos em que as decisões são tomadas (Manning, 1977, 2008). Este aspecto dos objetivos organizacionais é frequentemente alvo de debate.

A indeterminabilidade de tais contextos leva a fenômenos sócio-organizacionais que objetivam reduzir tais ambiguidades (Baran \& Scott, 2010). Uma forma de compreendê-los é por meio da noção de sensemaking, entendida como uma forma de criar sentido classificando em categorias os fluxos de experiências (Weick, Sutcliffe, \& Obstfeld, 2005).

Combater o crime e aplicar a lei são dois objetivos organizacionais das polícias de todo o mundo. Este escopo de atuação é influenciado pelas culturas organizacionais e ocupacionais dessas instituições 
(Chan, 1997; Gowricharn \& Çankaya, 2017; Ingram, Terrill, \& Paoline, 2018; Reiner, 2016; Turnbull \& Wass, 2015). Isto é, combater o crime depende de conteúdos simbólicos compartilhados entre os policiais. Porém a dimensão simbólica de combate ao crime tende a limitar a reação das polícias à pandemia, pois, o policial, costumeiramente, tem como sentido construído encarar o risco, diferentemente daquilo que deveria ser feito para lidar com o vírus (Alcadipani, 2020; Alcadipani et al., 2020). Logo, a situação caótica decorrente da pandemia exige novos enquadramentos para dar sentido aos processos e ações organizacionais.

Outros países, onde a COVID-19 se manifestou primeiramente, servindo-se da capilaridade da polícia, adicionaram funções extras ao seu trabalho, como: coleta de material para exames; estruturação de logísticas para movimentação de recursos específicos; levantamento de dados para a elaboração de ações estratégicas.

Segundo Weick et al. (2005), o sensemaking é um processo interacional e cognitivo que começa com o caos, mas que possibilita transformar as circunstâncias em balizas para as ações organizacionais. No contexto policial, estamos tratando dos processos de negociação da realidade sócio-organizacional (Manning, 2007). Além de o clima misto de incerteza, insegurança e desorientação já estar relacionado com a natureza extrema do trabalho policial (Granter, McCann, \& Boyle, 2015), a pandemia traz aspectos novos, sobretudo ligados à dimensão biológica do contágio e da disseminação do vírus, dimensão essa que não pode ser ignorada no estabelecimento de sentidos para o funcionamento das polícias.

Os desafios trazidos pelo cenário atual exigem que as forças policiais compreendam que se trata de um momento de ação humanitária, no qual o recurso à força deixa de ser protagonista dos modos de atuação para dar lugar à construção de uma governabilidade negociada. Para que os policiais atuantes nas ruas se enquadrem a este novo perfil, são necessários dispositivos, o que pode ser entendido como o encadeamento preparado de sequências, objetivando qualificar ou transformar estados de coisas pelo agenciamento de elementos materiais e linguísticos (Dodier \& Barbot, 2016). Elementos materiais correspondem aos processos e aos documentos administrativos que auxiliam e balizam a tomada de decisões dos "burocratas em nível de rua" (Lipsky, 2010), além de equipamentos de proteção específicos; e elementos linguísticos, o conjunto de termos e narrativas baseado em expertises da saúde, especialmente necessárias no momento. Isto é, em momentos caóticos, como o atual, as organizações e suas lideranças devem criar dispositivos que auxiliem os sujeitos a darem sentido às situações e aos contextos. Além do mais, é fundamental que os policiais sejam capacitados e entendam os riscos assumidos e a importância do seu trabalho na superação desta crise, por meio de fluxos de informações claras, fundamentadas cientificamente (Djalante, Shaw, \& DeWit, 2020).

Considerando-se o caráter humanitário que as normas do funcionamento das organizações e da tomada de decisões devem adotar, é importante que as organizações criem elementos linguísticos e materiais que delineiem claramente os contextos, tendo em vista influenciar na formação de sentido dos seus membros. Em termos práticos, padrões e protocolos de atendimentos devem ser elaborados, sem deixar de se levar em conta a situação atípica dos infectados assintomáticos (Zhou et al., 2020). No plano administrativo-burocrático, ações devem garantir, por exemplo, orçamento para a disponibilização de materiais de proteção individual e coletiva nas unidades.

Dito isso, o sensemaking não significa produzir uma narrativa única daquilo que está acontecendo, mas apresenta pontos de apoio convencionais para o desenvolvimento das ações. Várias condicionantes influenciam este processo, sendo o papel das lideranças fundamental, principalmente, em organizações 
policiais, dadas as suas características hierárquico-organizacionais. Desenvolveremos este aspecto na última parte do artigo.

\section{DISCRICIONARIEDADE E EMOÇÃO: POLÍCIAS EM TEMPOS DE PANDEMIA}

As experiências são irredutíveis (James, 1904), ou seja, elas não podem ser consideradas predominantemente por um único aspecto, seja ele cognitivo ou sensorial. Considerar os processos supracitados como eminentemente racionais, apartando-os das dimensões corpórea e emocional, trata-se, ao nosso ver, de um erro, sobretudo no momento atual. As emoções acontecem nos corpos. São eles, portanto, dimensões da criação de sentido, resultando na influência das emoções sobre os processos de sensemaking (Maitlis, Vogus, \& Lawrence, 2013). Desse modo, os estados corporais afetam e são afetados pela situação; além do mais, as pesquisas sobre cognição incorporada demonstram que os processos cognitivos são fundamentados nos corpos (Hernes \& Maitlis, 2010; Maitlis \& Sonenshein, 2010). Em se tratando das polícias, todas as questões levantadas devem ser pensadas relativamente ao corpo, elemento importante da identidade do trabalho policial (Courpasson \& Monties, 2017).

Em face do acima exposto, o corpo, no caso da pandemia, passa a receber uma carga valorativa: ele é tanto a dimensão pela qual há proliferação do vírus e contágio da doença, o que, portanto, requer cuidados específicos, como é por meio dele e das emoções - que serão postas cada vez mais à prova em virtude da doença que atingirá o próprio policial, seus companheiros de trabalho e familiares que os arranjos institucionais necessitam ser construídos. Por tais razões, as organizações carecem apresentar preocupações concernentes à saúde física e mental no trabalho, sobretudo devido às crescentes taxas de contaminação que promoverão diminuição na força de trabalho.

Para complementar, não se podem criar discursos que encorajem o trabalho a qualquer custo; um policial com suspeita de contaminação (ou que resida com alguém suspeito) precisa atender estritamente às recomendações das organizações de saúde e ser afastado do trabalho, caso contrário, ele representa um risco para os demais integrantes da polícia e também para os seus familiares. $\mathrm{O}$ índice de contágio da doença é alto (Zhou et al., 2020), e a exposição irresponsável dos policiais ao contágio pode esvaziar regiões inteiras de seus contingentes policiais ativos.

Muito embora se esteja tratando de organizações com trabalhos com alto nível de estresse (Granter et al., 2015), é importante destacar que esta crise não tem precedentes. Logo, muitos provavelmente serão infectados, o que leva à diminuição dos contingentes e aumenta o trabalho cotidiano ligado às estabelecidas tarefas de enfrentamento ao crime e atendimento aos chamados de emergência, pois, em tempos como este, de reconfiguração dos fluxos de pessoas e de capitais, novas formas de conflitualidade e de crimes tendem a surgir, estabelecendo novos desafios às corporações.

Os policiais são burocratas em nível de rua, ou seja, trabalham diretamente com os cidadãos e contam com uma substancial discrição em suas atividades (Lipsky, 2010). Tal discricionariedade é, frequentemente, tratada com desconfiança por parte das estruturas e instâncias de controle (Cavalcanti, Lotta, \& Pires, 2018). O espaço de discricionariedade se refere às perspectivas organizacionais, bem como à relação entre superiores e subordinados; já a ação de discricionariedade se dá pela forma como os burocratas compreendem os conteúdos normativos (Lotta \& Santiago, 2017).

Diante deste cenário, é fundamental que o corpo diretivo das organizações policiais mapeie e, quando possível, delineie o espaço de discricionariedade a fim de garantir segurança administrativa e jurídica às tomadas de decisão em nível local. A obtenção de materiais deve ser realizada, sempre

REVISta de Administração Pública | Rio de Janeiro 54(4):898-908, jul. - ago. 2020 
que possível, pelas estruturas organizacionais diretamente envolvidas no enfrentamento da pandemia. Pesquisas no setor demonstram que ao invés de tais estruturas organizacionais efetuarem tal tarefa, há organismos burocráticos intermediários para este fim, por exemplo, os centros responsáveis pelas compras, unidades regionais, seccionais e estruturas específicas. As justificativas para este cenário de centralização se dão pela maior possibilidade de controle. No entanto, os atores relatam sobre a falta de celeridade. Nesse sentido, entendemos ser necessário a descentralização da gestão de parte do orçamento para a garantia do atendimento das organizações e dos policiais.

\section{POLÍCIAS E PANDEMIA: 0 QUE FAZER?}

Com base no que foi exposto, perguntamos: o que as polícias brasileiras podem fazer em um cenário de pandemia? Emergências públicas requerem rápida adaptação às condições instáveis, gestão da distribuição da informação, coordenação bilateral e ação coletiva emergente (Nowell, Steelman, Velez, \& Yang, 2018). No Brasil, a falta de coordenação política contribui para a descentralização do trabalho das polícias no combate ao vírus, e impõe constrições políticas, materiais e culturais que imobilizam as corporações e prejudicam seu trabalho com a população (Alcadipani et al., 2020; Lotta et al., 2020).

Experiências de outros países demonstram a necessidade de articulação interorganizacional a fim de que as polícias mobilizem-se para o enfrentamento da pandemia, seja pela criação de mecanismos de coordenação ou pelo acionamento dos já existentes (Djalante et al., 2020; Legido-Quigley et al., 2020; Nkengasong \& Mankoula, 2020; Zhao et al., 2020). O Brasil possui um Plano de Resposta em Emergências em Saúde Pública (Ministério da Saúde, 2014), oriundo do Ministério da Saúde do governo federal, elaborado para a coordenação dos esforços em caso de crises de saúde pública em âmbito nacional e internacional. O plano prevê a ativação de um Centro de Emergência em Saúde Pública (COES) para "[...] promover a resposta coordenada por meio da articulação e da integração dos atores envolvidos" (Ministério da Saúde, 2014, p. 14), com a instituição prévia de protocolos de ação para estes atores. Contudo essa articulação contempla apenas os atores ligados ao âmbito da saúde, sem que a promoção de enforcement tenha sido cotejada. Este plano difere, por exemplo, do norte-americano, que, em casos de ameaça à saúde pública, dispõe de instrumentos institucionais de coordenação de forças policiais com as autoridades sanitárias (Richards, Rathburn, Brito, \& Luna, 2006).

Apontamos, assim, para a necessidade de aprofundamento do Plano de Respostas em Emergências em Saúde Pública, dotando-o de uma amplitude que alcance o trabalho policial nas medidas sanitárias. Tal cenário possibilita uma maior capacidade de coordenação nacional dos esforços, tem o potencial de atender os problemas suscitados neste trabalho, ao conferir novo sensemaking aos arranjos policiais locais e garantir aspectos de constituição de uma legitimidade necessária às ações que são demandadas, reorientando as práticas policiais já consolidadas em seu cotidiano. Todavia esta é uma ação de longo prazo, que mobilizaria muitos atores políticos, os quais, no Brasil, encontram-se em posição de divergência e conflito.

No curto prazo, vale observarmos os exemplos de outras polícias do mundo, considerando a pesquisa "A pandemia da COVID-19 e os policiais brasileiros" (2020) que mostra que 43,9\% dos policiais não se sente preparado e $24,5 \%$ não soube responde como atuar na pandemia (Lotta et al., 2020). Na Índia, numa atitude de caráter humanitário, a polícia vem tentando ressignificar a sua relação com as pessoas, atuando no auxílio às vítimas da pandemia e no atendimento à população em geral (Gettleman, Singh, Kim, \& Kumar, 2020). Ao redor do mundo e no Brasil, inúmeros departamentos de 
polícia instituíram protocolos regulamentando como os policiais devem abordar suspeitos, interrogar testemunhas e transportar presos. O College of Policing do Reino Unido e a Interpol têm compartilhado guias e protocolos específicos para a atuação policial durante a pandemia, que abordam diferentes dimensões do trabalho. A realização de vídeos curtos mostrando os protocolos na prática podem ser uma forma rápida de difusão da utilização de equipamentos de proteção individual, como tem sido feito pela Polícia Militar de São Paulo (PMTV, 2020).

Desta lógica, é fundamental a identificação de policiais com sintomas que possam estar infectados, para evitar a contaminação de outros agentes e de seus familiares. Com a realização de testes rápidos em todos os profissionais das polícias, é possível identificar aqueles que tiveram contato com o vírus e que, por esta razão, precisam ser afastados de suas atividades. Todavia a pandemia trouxe o problema da escassez de testes no mundo todo (Ranney, Griffeth, \& Jha, 2020). A impossibilidade de acesso aos testes e a contaminação dos agentes, posteriormente à sua realização, tornam necessárias medidas complementares, como a verificação dos níveis de oxigênio e da temperatura de todos os policiais que chegam ao trabalho, como já tem sido feito nos EUA e na Europa. A identificação de um policial contaminado requer o seu isolamento por 14 dias. Em alguns locais, hotéis foram utilizados, pois, caso haja o agravamento da doença, o policial pode ser atendido prontamente, evitando-se, ao mesmo tempo, a transmissão do vírus para sua família.

É importante, ainda, que as corporações pratiquem, tanto quanto possível, o distanciamento social entre os policiais nas unidades e entre os policiais e o público; priorizem o atendimento de urgências e o patrulhamento, mas seguindo protocolos específicos que determinem quando a abordagem policial deve ser realizada. Reorganizar as tarefas para que a interação com a população seja feita virtualmente é uma importante medida de proteção aos policiais. Em São Paulo, a Polícia Civil passou a registrar online uma maior variedade de crimes e também elaborou uma delegacia eletrônica da mulher com atendimento feito por alunos em formação na Academia de Polícia. A lógica deveria ser a de que os policiais fossem para os seus locais de trabalho somente quando necessário. Além disso, há polícias fora do Brasil que criaram forças tarefas de policiais destinadas a cobrir os afastamentos de colegas pela doença. É importante suspender todas as cerimônias presenciais das instituições, como adotado pela Polícia Militar de São Paulo. Ainda, há polícias que estão alterando os turnos de trabalho tendo como objetivo auxiliar no distanciamento social e, com isso, diminuir o número de pessoas compartilhando espaços ao mesmo tempo.

O enfrentamento de crises salienta os papéis das lideranças organizacionais, já que exercem influência sobre as condições definidoras das situações e as formas de compreendê-las (Whittle, Housley, Gilchrist, Mueller, \& Lenney, 2015), bem como auxiliam no processo de produção de sentidos dentro das organizações. Estudos indicam que a criação de sentido por parte dos líderes é um processo que nem sempre atende à velocidade dos fatos vividos; por certo, a formação de consenso e a sensibilização dos indivíduos envolvidos exigem um certo tempo (Combe \& Carrington, 2015). A discussão sobre o papel das lideranças é ainda mais fundamental em organizações policiais, as quais são fundadas em hierarquias.

As lideranças organizacionais e políticas devem afinar seus discursos e buscar fundamentos em campos de expertises mais consolidadas, corroborados pela técnica e pela ciência ("Coronavirus: three things all governments and their science advisers must do now”, 2020). Assim, as lideranças encontrarão mais condições de promover novos contextos adaptativos às necessidades que surgirão com o desenvolvimento da pandemia, embasando as mudanças organizacionais. Sobre isso, a profusão 
de informações falsas (fakenews) e/ou de pouca credibilidade nas redes sociais cria dificuldades para as lideranças e para a construção cognitiva de novos ambientes. Dessa forma, o papel das lideranças é importante para nortear os enquadramentos, sem dúvidas ou confusões acerca das ações. As polícias que estão respondendo melhor ao desafio são aquelas cujas lideranças internas assumiram o papel de protagonismo: primeiramente, reconhecendo a gravidade da doença para as forças policiais e a necessidade de trabalhar de forma diferente diante da pandemia, e, depois, dando o exemplo da adoção de protocolos de segurança e comunicando frequentemente aos policiais qual o caminho a ser seguido. Desse modo, as lideranças policiais precisam deixar claro que o momento da pandemia da COVID-19 é excepcional: nesta situação apenas as urgências policiais devem mobilizar esforços de suas instituições, o distanciamento social deve ser levado a sério dentro das organizações policiais. Além disso, seus líderes precisam direcionar suas organizações para que estas trabalhem muito mais como instituições de apoio humanitário do que como instrumentos de combate ao crime.

\section{CONSIDERAÇÕES FINAIS}

A pandemia da COVID-19 parece constituir o maior desafio encontrado pela humanidade desde a Segunda Guerra Mundial. Ela nos proporciona a experiência da incerteza do mundo e da nossa relação com a natureza (Dewey, 1958; James, 1904). Essas questões colidem brutalmente com as lógicas de funcionamento das organizações, principalmente no que concerne às perspectivas da racionalidade, dos objetivos e dos sentidos construídos organizacionalmente.

Neste artigo, procuramos evidenciar que o trabalho das polícias deve ser alterado para o enfrentamento da pandemia mundial, o que requer esforços no sentido de que as organizações policiais sejam adicionadas às coordenações interagências. Para tanto, pontuamos que as estruturas mais capilares dessas organizações necessitam de apoios variados.

Todavia, além da questão de gestão de poder na organização, importam os sentidos que orientam as mudanças necessárias, e a construção de novos dispositivos materiais e linguísticos que promovam novos enquadramentos de atuação das polícias. É necessário que as organizações policiais mudem suas lógicas de funcionamento, passando da noção de "guerra contra o crime" para a de ações humanitárias.

Assim, é imperioso que as organizações se afinem por meio de um mesmo plano informacional, apresentando narrativas consonantes entre si, desenvolvendo métodos e sentidos organizacionais que estabeleçam a segurança pública como uma ação humanitária.

As emoções e a discricionariedade são aspectos a serem considerados, especialmente, pelos profissionais que atuam na linha de frente de combate à pandemia. Acreditamos, assim, que essas organizações se constituirão como ferramentas de enfrentamento desta crise e possam ver nesta situação uma janela de oportunidade para estabelecer novas relações com seus públicos. Conforme demonstra o caso da Índia, atuar promovendo ação humanitária pode aproximar a polícia da sociedade, elemento fundamental para o bom funcionamento de qualquer política de segurança pública. 


\section{REFERÊNCIAS}

Alcadipani, R. (2020). Pandemic and macho organizations: wakeup call or business as usual? Gender, Work \& Organization, Early view, 1-13. Recuperado de https://doi.org/10.1111/gwao.12466

Alcadipani, R., Cabral, S., Fernandes, A., \& Lotta, G. (2020, June). Street-Level bureaucrats under COVID-19: police officers' responses in constrained settings. Administrative Theory \& Praxis, 1-10. Recuperado de https://doi.org/10.1080/10841806. 2020.1771906

Baran, B. E., \&Scott, C. W. (2010, January). Organizing ambiguity: a grounded theory of leadership and sensemaking within dangerous contexts. Military Psychology, 22 (Suppl. 1), S42-S69. Recuperado de https://doi.org/10.1080/08995601003644262

Cavalcanti, S., Lotta, G. S., \& Pires, R. R. C. (2018). Contribuições dos estudos sobre burocracia de nível de rua. In R. Pires, G. S. Lotta, \& V. E. Oliveira (Orgs.), Burocracia e políticas públicas no Brasil: interseções analíticas (pp. 227-246). Brasília, DF: Ipea/ Enap. Recuperado de http://repositorio.enap.gov. br/bitstream/1/3247/1/livro_Burocracia e políticas públicas no Brasil - interseções analíticas.pdf

Chan, J. B. L. (1997). Changing police culture. Policing in a multicultural society. Cambridge, UK: Cambridge University Press.

Combe, I. A., \& Carrington, D. J. (2015). Leaders' sensemaking under crises: emerging cognitive consensus over time within management teams. Leadership Quarterly, 26(3), 307-322. Recuperado de https://doi.org/10.1016/j.leaqua.2015.02.002

Comes, T. (2016, March). Cognitive biases in humanitarian sensemaking and decision-making lessons from field research. Proceedings of 2016 IEEE International Multi-Disciplinary Conference on Cognitive Methods in Situation Awareness and Decision Support, (pp. 56-62), San Diego, CA. Recuperado de https://doi.org/10.1109/ COGSIMA.2016.7497786

Coronavirus: three things all governments and their science advisers must do now. (2020, March 17). Nature, 579(7799), 319-320. Recuperado de https:// doi.org/10.1038/d41586-020-00772-4
Courpasson, D., \& Monties, V. (2017). "I am my body". Physical selves of police officers in a changing institution. Journal of Management Studies, 54(1), 32-57. Recuperado de https://doi.org/10.1111/ joms. 12221

Dewey, J. (1958). Experience and nature. New York, NY: Dover.

Djalante, R., Shaw, R., \& DeWit, A. (2020, April). Building resilience against biological hazards and pandemics: COVID-19 and its implications for the Sendai Framework. Progress in Disaster Science, 6, 100080. Recuperado de https://doi.org/10.1016/j. pdisas.2020.100080

Dodier, N., \& Barbot, J. (2016). La force des dispositifs. Annales. Histoire, Sciences Sociales, 71(02), 421-448. Recuperado de https://doi. org/10.1353/ahs.2016.0064

Gettleman, J., Singh, K. D., Kim, C., \& Kumar, H. (2020, June 01). How India's Police Used a Pandemic to Boost Its Image. NYTIMES. Recuperado de https://www.nytimes.com/video/ world/100000007141490/-india-police-pandemiccoronavirus.html?action $=$ click\&gtype $=$ vhs \&ver sion $=$ vhs - heading \&module $=$ vhs \&region $=$ title area $\&$ cview $=$ true $\& \mathrm{t}=4$

Giacomantonio, C. (2014). A typology of police organizational boundaries. Policing and Society, 24(5), 545-565. Recuperado de https://doi.org/10.1 080/10439463.2013.784302

Gowricharn, R., \& Çankaya, S. (2017). Policing the Nation: acculturation and streetlevel bureaucrats in professional life. Sociology, 51(5), 1101-1117. Recuperado de https://doi. org/10.1177/0038038515601781

Granter, E., McCann, L., \& Boyle, M. (2015). Extreme work/normal work: intensification, storytelling and hypermediation in the (re)construction of 'the New Normal.' Organization, 22(4), 443-456. Recuperado de https://doi.org/10.1177/1350508415573881

Hernes, T., \& Maitlis, S. (2010). Process, sensemaking, and organizing: an introduction. In T. Hernes \& S. Maitlis (Eds.), Process, Sensemaking, \& Organizing (Perspectiv, pp. 27-37). New York, NY: Oxford University Press.

Ingram, J. R., Terrill, W., \& Paoline, E. A. (2018). Police culture and officer behavior: application of 
a multilevel framework. Criminology, 56(4), 780811. Recuperado de https://doi.org/10.1111/17459125.12192

James, W. (1904). The world of pure experience. The Journal of Philosophy, Psychology and Scientific Methods, 1(20), 533-543.

Latour, B. (2012). Reagregando o social: uma introdução à teoria do Ator-Rede. Salvador, BA: EDUFBA; EDUSC.

Legido-Quigley, H., Mateos-García, J. T., Campos, V. R., Gea-Sánchez, M., Muntaner, C., \& McKee, M. (2020). The resilience of the Spanish health system against the COVID-19 pandemic. The Lancet Public Health, 5(5), e251-e252. Recuperado de https://doi. org/10.1016/S2468-2667(20)30060-8

Lipsky, M. (2010). Street-level democracy: dilemmas of the individual in public services (30th anniv.). New York, NY: Russell Sage Foundation.

Lotta, G., \& Santiago, A. (2017). Autonomia e discricionariedade: matizando conceitos-chave para o estudo da burocracia. BIB, 1(83), 21-42.

Lotta, G., Sobral, I., Corrêa, M., Alcadipani, R., \& Bueno, S. (2020, 18 de maio). A pandemia de Covid-19 e os policiais brasileiros (Nota técnica). São Paulo, SP: Fórum Brasileiro de Segurança Pública. Recuperado de https://forumseguranca.org.br/ publicacoes_posts/a-pandemia-de-covid-19-e-ospoliciais-brasileiros/

Maitlis, S., \& Sonenshein, S. (2010). Sensemaking in crisis and change: inspiration and insights from Weick (1988). Journal of Management Studies, 47(3), 551-580. Recuperado de https://doi.org/10.1111/ j.1467-6486.2010.00908.x

Maitlis, S., Vogus, T. J., \& Lawrence, T. B. (2013). Sensemaking and emotion in organizations. Organizational Psychology Review, 3(3), 222-247. Recuperado de https://doi. org/10.1177/2041386613489062

Manning, P. K. (1977). Police work: the social organization of policing. Cambridge, UK: MIT Press.

Manning, P. K. (2007). A dialectic of organisational and occupational culture. In M. O'Neill, M. Marks, \& A.-M. Singh (Eds.), Police occupational culture: new debates and directions (pp. 47-84). Oxford, UK: JAI Press. Recuperado de https://doi.org/10.1016/ S1521-6136(07)08002-5
Manning, P. K. (2008). The technology of policing: crime mapping, information technology, and the rationality of crime control. New York, NY: New York University Press.

Mastrofski, S. D., \& Willis, J. J. (2010). Police organization continuity and change: into the twenty-first century. Crime and Justice, 39, 55-144. Recuperado de https://doi.org/10.1086/653046

Ministério da Saúde. Secretaria de Vigilância em Saúde. Departamento de Vigilância em Saúde Ambiental e Saúde do Trabalhador. (2014). Plano de Resposta às Emergências em Saúde Pública. Brasília, DF: Autor.

Monjardet, D. (1998). Elementos de análisis de los sistemas policiales. Revista Catalana de Seguretat Pública, 2, 179-194.

Nkengasong, J. N., \& Mankoula, W. (2020). Looming threat of COVID-19 infection in Africa: act collectively, and fast. The Lancet, 395(10227), 841-842. Recuperado de https://doi.org/10.1016/ S0140-6736(20)30464-5

Nowell, B., Steelman, T., Velez, A. L. K., \& Yang, Z. (2018). The structure of effective governance of disaster response networks: insights from the field. American Review of Public Administration, 48(7), 699-715. Recuperado de https://doi. org/10.1177/0275074017724225

PMTV. (2020, 17 de abril). Atuação da PMESP em tempos de COVID-19. Youtube. Recuperado de https://www.youtube.com/watch?v=T2Xn21xowP0

Quarantelli, E. L. (1988). Disaster crisis management. Journal of Management Studies, 25(4), 373-385. Recuperado de https://doi. org/10.1111/j.1467-6486.1988.tb00043.x

Ranney, M. L., Griffeth, V., \& Jha, A. K. (2020). Critical supply shortages - the need for ventilators and personal protective equipment during the Covid-19 pandemic. New England Journal of Medicine, 382(18), e41(1)-e41(3). Recuperado de https://doi.org/10.1056/NEJMp2006141

Reiner, R. (2016, December 05). Is police culture cultural? Policing, (Opinion), 11(3), 236-241. Recuperado de https://doi.org/10.1093/police/ paw046 
Richards, E. P., Rathburn, K. C., Brito, C. S., \& Luna, A. (2006). The Role of Law Enforcement in Public Health Emergencies. Washington, DC: U.S. Department of Justice.

Rubinstein, J. (1973). City Police. New York, NY: Farrar, Straus and Giroux.

Stephenson Jr., M. (2005). Making humanitarian relief networks more effective: operational coordination, trust and sense making. Disasters, 29(4), 337-350. Recuperado de https://doi.org/10.1111/j.03613666.2005.00296.x

Turnbull, P. J., \& Wass, V. (2015). Normalizing extreme work in the Police Service? Austerity and the inspecting ranks. Organization, 22(4), 512-529. Recuperado de https://doi. org/10.1177/1350508415572513

Weick, K. E., Sutcliffe, K. M., \& Obstfeld, D. (2005). Organizing and the Process of Sensemaking.
Organization Science, 16(4), 409-421. Recuperado de https://doi.org/10.1287/orsc.1050.0133

Whittle, A., Housley, W., Gilchrist, A., Mueller, F., \& Lenney, P. (2015). Category predication work, discursive leadership and strategic sensemaking. Human Relations, 68(3), 377-407. Recuperado de https://doi.org/10.1177/0018726714528253

Zhao, W., Zhang, J., Meadows, M. E., Liu, Y., Hua, T., \& Fu, B. (2020, June 15). A systematic approach is needed to contain COVID-19 globally. Science Bulletin, 65(11), 876-878. Recuperado de https:// doi.org/10.1016/j.scib.2020.03.024

Zhou, F., Yu, T., Du, R., Fan, G., Liu, Y., Liu, Z., ... Cao, B. (2020, March 09). Clinical course and risk factors for mortality of adult inpatients with COVID-19 in Wuhan, China: a retrospective cohort study. The Lancet, 395, 1054-1055. Recuperado de https://doi.org/10.1016/S01406736(20)30566-3

\section{Gustavo Matarazzo}

https://orcid.org/0000-0002-4640-3863

Pós-doutorando em Administração pela Escola de Administração de Empresas de São Paulo (EAESP) da Fundação Getulio Vargas (FGV); Professor do Instituto Federal de Educação, Ciência e Tecnologia de São Paulo (IFSP) Campus Capivari. E-mail: gustavo.matarazzo@ifsp.edu.br

\section{Alan Fernandes}

https://orcid.org/0000-0003-1364-3868

Doutorando em Administração pela Escola de Administração de Empresas de São Paulo (EAESP) da Fundação Getulio Vargas (FGV); Major na Polícia Militar do Estado de São Paulo. E-mail: alan@policiamilitar.sp.gov.br

\section{Rafael Alcadipani}

https://orcid.org/0000-0001-5132-5380

Doutor em Management Sciences pela Machester Business School; Professor titular da Escola de Administração de Empresas de São Paulo (EAESP) da Fundação Getulio Vargas (FGV). E-mail: rafael.alcadipani@fgv.br 